\title{
Quantification Et Evolution Du Bilan De La Nappe Karstique De Saida (Nord-Ouest De l'Algerie)
}

\author{
Medjber Abdellah \\ Université Dr Moulay Tahar de Saida (Algérie)
}

Faculté de Technologie, département de génie civil et hydraulique laboratoire des ressources hydriques et environnement, Hai Nasr, Saida, Algérie

\section{Berkane Fatiha}

Ingénieur Hydraulique, Direction de L'hydraulique, Saida, Algérie

\begin{abstract}
The Northwest of Algeria, characterized by a semi-arid climate has seen in recent decades its surface and ground water potential decrease due to the scarcity of rainfall. Demographic pressure has increased the difficulties in the management of water resources, leading to problems of water scarcity in several areas of the country, especially in low-rainfall areas. From the hydrogeological point of view, the study area contains many karstic springs with great importance, which flows can exceed $50 \mathrm{l} / \mathrm{s}$ during flood periods. Aquifer recharge occurs primarily by rainwater.The main objective of this study was to evaluate the water balance of the karst aquifer situated near the city of Saida (North-West of Algeria) and to follow its evolution on several decades. In this region, the karst groundwater is important for drinking water supply, irrigation and industrial units.This approach to assessment will be based on the water balance equation by involving precipitation, evapotranspiration, infiltration and groundwater flow. The total volume of the groundwater reserves obtained from the balance method represent 45 million $\mathrm{m} 3$. These reserves have decreased with time.
\end{abstract}

Keywords: Water balance, karst groundwater, precipitation, groundwater flow, watershed .

\section{Résumé}

Le Nord-Ouest Algérien caractérisé par un climat semi-aride a vu ces dernières décennies ses potentialités en eau superficielles et souterraines diminuer du fait de la rareté des précipitations. La pression démographique a augmenté les difficultés dans la gestion des ressources en eau, raison pour laquelle les problèmes de pénurie d'eau apparaissent dans plusieurs secteurs 
du pays, surtout ceux qui connaissent des apports pluviométriques très bas. Du point de vue hydrogéologique, la région étudiée renferme des sources karstiques de grande importance, avec des débits qui dépassent $50 \mathrm{l} / \mathrm{s}$ en périodes de crue. La recharge de la nappe se fait essentiellement par les eaux de pluie. L'objectif principal de l'étude est d'évaluer le bilan hydrique de la nappe karstique de la région de Saida (Nord-Ouest de l'Algérie) et de suivre son évolution au cours des dernières décennies, nappe qui est utilisée pour l'alimentation en eau potable de la ville de Saida mais aussi pour l'irrigation et les unités industrielles. Cette approche du bilan sera basée sur l'équation $\mathrm{du}$ bilan hydrique en faisant intervenir les précipitations, l'évapotranspiration, l'infiltration et les écoulements souterrains. Le volume total des réserves obtenu à partir de cette méthode du bilan nous donne une valeur de 45 millions de $\mathrm{m}^{3}$. Ces réserves ont diminuées avec le temps.

Mots clés : Bilan, nappe karstique, précipitations, écoulements souterrains, bassin versant

\section{Situation Geographique De La Region}

La zone étudiée se situe au Nord-Ouest de l'Algérie, Elle se trouve à environ $180 \mathrm{Km}$ au Sud d'Oran dans la zone des Hauts plateaux, l'altitude moyenne de cette région est de $900 \mathrm{~m}$.

Cette région se caractérise par un climat de type semi aride avec de très rares oueds pérennes.

Cette position lui donne un rôle de relais entre les régions steppiques au Sud et celles telliennes au Nord.

Ce territoire s'étend sur deux domaines naturels bien distincts, l'un est atlasique Tellien au Nord et l'autre est celui des hautes plaines steppiques au Sud.

Elle est limitée naturellement au Sud par le chott Chergui, au nord par la wilaya de Mascara, au sud par celle d'El Bayadh, à l'est par la wilaya de Tiaret et à l'ouest par la wilaya de Sidi- bel -Abbès (figure 1).

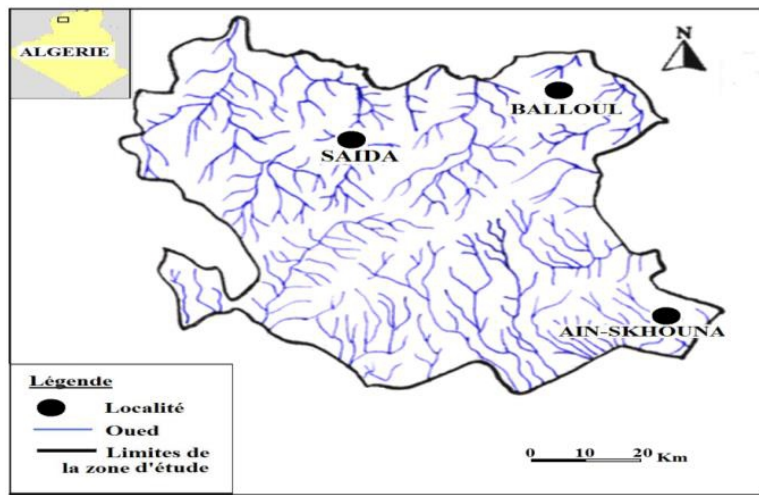

Figure 1. Carte de situation de la région d'étude 


\section{Geologie de la region}

La zone d'étude est caractérisée par une géologie complexe et diversifiée, représentée par des terrains allant du Primaire au PlioQuaternaire.

Elle constituée principalement de formations du Jurassique (dolomies, argiles gréseuses, marnes, calcaires ...) avec quelques roches éruptives et des formations d'âge primaire; les dépressions sont constituées par des alluvions du Quaternaire.

Les roches (principalement des dolomies et des calcaires) sont très karstifiées d'après Decamps (1973).

Elles sont recouvertes par l'épaisse formation « argilo gréseuse » du Callovo-Oxfordien au niveau de la vallée de l'Oued Saïda, et par endroits, par des formations non consolidées plus récentes (Quaternaire et PlioQuaternaire).
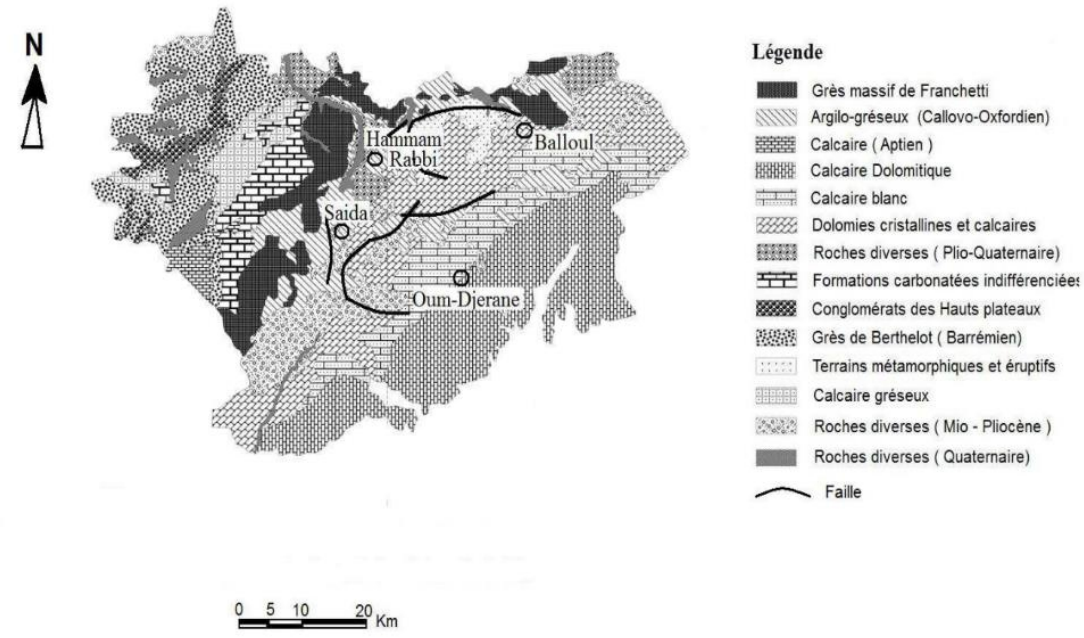

Figure 2. Carte géologique de la région de Saida

Les formations anciennes sont affectées par une tectonique de type alpine caractérisée par de grands plis donnant naissance aux Monts de Saïda, et de grandes failles de direction générale Sud-ouest et Nord-est dont les plus importantes limitant la ville de Saïda vers le côté Nord-Ouest (failles de Zeboudj) et le côté Sud-est. On trouve successivement du Nord au Sud : une chaîne alpine (Tell Occidental), une zone tabulaire (Haute plaine Oranaise), une chaîne tertiaire (Atlas Saharien) limitée au Sud par l'accident atlasique qui la sépare du bouclier Saharien.

Le karst de surface est représenté surtout par des dolomies et calcaires, (plateau de Tidernatine). Les dolomies sont nombreuses sur ce plateau et donnent naissance à des entonnoirs qui sont pénétrables et spectaculaires (Ghar Ouled - Amira, Ghar Slouguia ...). 
Le karst le plus profond de la région, le plus connu par les spéléologues est celui d'Ain -Zerga drainé par les rivières souterraine de Bir- Hamama et de Ghar Ouled - Amira (Est de la ville de Saida ). Ces rivières pourraient constituer le collecteur principal du système karstique du causse de Tidernatine.

Enfin, dans les dépressions ainsi que les vallées et lits d'Oueds, on trouve des terrains d'origine continentale (fluviatiles et éoliens) d'âge tertiaire souvent indifférenciés : Mio-Pliocène et Quaternaire. Le continental est également caractérisé par la présence de tufs et travertins plus ou moins récents et correspondants à d'anciens griffons de sources, Decamps (1973). La lithostratigraphie de la région est présentée en figure 3.

\begin{tabular}{|c|c|c|c|c|c|}
\hline \multicolumn{2}{|c|}{ Âge } & \multirow{2}{*}{ Log } & \multirow{2}{*}{\begin{tabular}{|l|}
$\begin{array}{l}\text { Epaiss- } \\
\text { eur }\end{array}$ \\
$20 \mathrm{~m}$ \\
\end{tabular}} & \multicolumn{2}{|c|}{ Description lithologique } \\
\hline & Quaternaire & & & \multicolumn{2}{|c|}{ Limons plus ou moins schisteux et travertins } \\
\hline & Tertiaire & 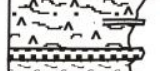 & $75 \mathrm{~m}$ & \multicolumn{2}{|c|}{$\begin{array}{l}\text { Argiles briques sableuses ou gypseuses } \\
\text { intercalés de calcaires et de graviers ou galets }\end{array}$} \\
\hline \multirow{2}{*}{ 造 } & Sénonien & & $100 \mathrm{~m}$ & \multicolumn{2}{|c|}{$\begin{array}{l}\text { Calcaires gris clairs à pâte fine très altérés } \\
\text { à la partie superficielle }\end{array}$} \\
\hline & Barrémien & & 30 à & \multirow{2}{*}{\multicolumn{2}{|c|}{ 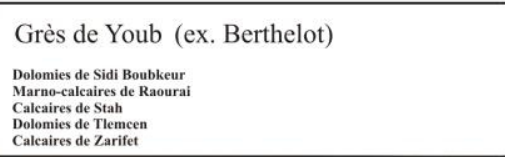 }} \\
\hline \multirow{6}{*}{ 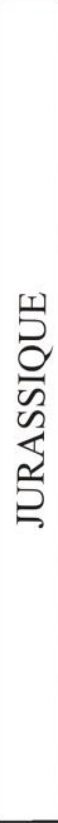 } & Kimméridjien & 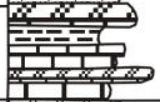 & $30 \mathrm{~m}$ & & \\
\hline & Lusitanien & 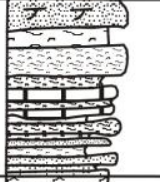 & $180 \mathrm{~m}$ & \multicolumn{2}{|c|}{$\begin{array}{l}\text { Grès de Sidi Amar (ex. Franchetti) } \\
\text { avec de rares passées carbonatées et } \\
\text { des argiles sableuses. }\end{array}$} \\
\hline & Callovo-oxfordien & $=1$ & $\begin{array}{l}180 \mathrm{à} \\
350 \mathrm{~m}\end{array}$ & \multicolumn{2}{|c|}{$\begin{array}{l}\text { Argiles de Saida : série argilo-grèseuse } \\
\text { à dominance argileuse }\end{array}$} \\
\hline & Aaléno-bajo-bathonien & 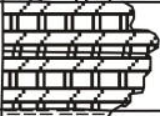 & $\begin{array}{l}110 \text { à } \\
150 \mathrm{~m}\end{array}$ & \multicolumn{2}{|c|}{$\begin{array}{l}\text { Dolomies supérieures ou Calcaires de } \\
\text { Balloul ; Dolomies cristallines } \\
\text { et calcaires fissurés }\end{array}$} \\
\hline & Toarcien & 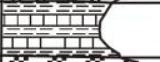 & $\begin{array}{l}15 \grave{a} \\
25 \mathrm{~m}\end{array}$ & \multicolumn{2}{|c|}{ Marno-calcaires de Keskes } \\
\hline & Domérien & yxy & $\begin{array}{l}30 \mathrm{à} \\
50 \mathrm{~m}\end{array}$ & \multicolumn{2}{|c|}{$\begin{array}{l}\text { Dolomies de Tiffrit ou Dolomies inférieures: } \\
\text { Dolomies cristallines bréchiques avec argiles }\end{array}$} \\
\hline & Trias & 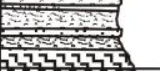 & & $\begin{array}{l}\text { Argiles salines, Grès- } \\
\text { argileux, Basalte }\end{array}$ & \multirow{2}{*}{$\begin{array}{l}\text { Complexe volcano- } \\
\text { sédimentaire }\end{array}$} \\
\hline & Primaire & (iv/ $/ 4$ & & $\begin{array}{l}\text { Schistes-granite- } \\
\text { rhyolites-diorites }\end{array}$ & \\
\hline
\end{tabular}

Figure 3. Log- stratigraphique de la région d'étude

Cadre hydrogeologique de la region et de la nappe karstique de saida Les études effectuées pour évaluer les ressources en eaux 
souterraines de la région ont pu mettre en évidence les nappes souterraines suivantes: (Figure 4).

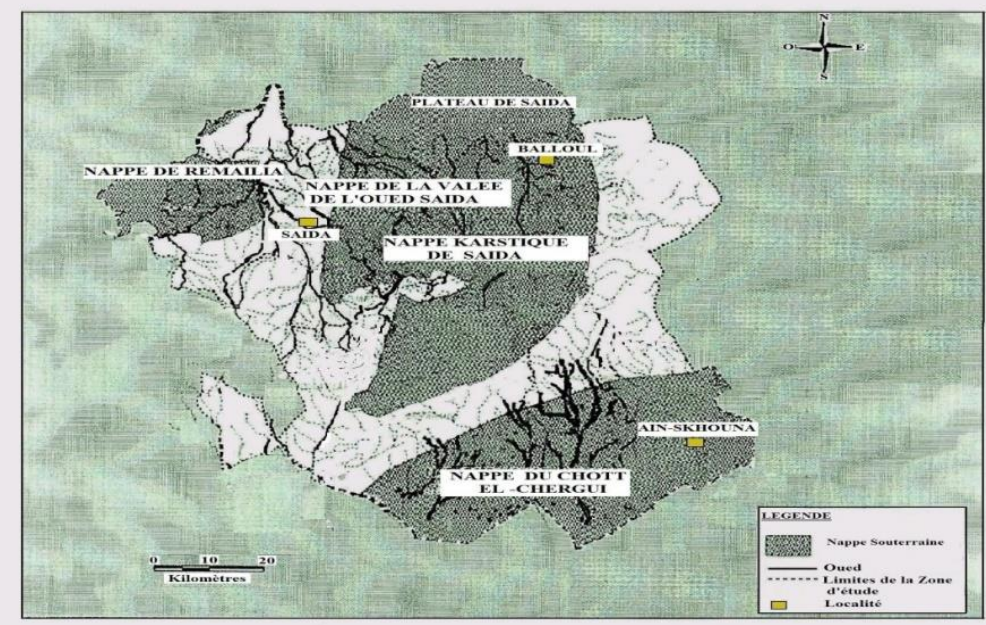

Figure 4. Répartition des nappes aquifères dans la région.

- Une nappe superficielle de la vallée de l'oued Saida généralement libre, située dans les horizons supérieurs de la formation de Saïda et les dépôts plio-quaternaires (sables argileux, argiles calcaires et conglomérats). D'une épaisseur de 10 à 15 mètres, la nappe renferme un potentiel de l'ordre de $5 \mathrm{hm}^{3} /$ an et couvre une superficie de $228 \mathrm{~km}^{2}$.

Elle est contenue dans les terrains détritiques du CallovoOxfordien et du Plio-Quaternaire.

Cette nappe est bien répartie dans la vallée de l'Oued Saïda, dont l'aquifère est formé par les couches les plus supérieures de la couverture plio-quaternaire et la formation argilo-gréseuse du callovo-oxfordien, constituant un réservoir commun, peu profond drainé par plusieurs exutoires sous forme de source (Ain -Tebouda, Ain S Cet aquifère superficiel couvrant toute la vallée de l'Oued Saïda, est alimenté par sa surface. idi Ali, Ain -Bourached ).

Selon Pitaud (1973), la nature argileuse de l'ensemble de ces terrains rendrait difficile toute tentative de mobilisation de cette eau.

- La nappe karstique de Saida contenue dans les sédiments carbonatés du jurassique inférieur et moyen. Ce réservoir karstique, constitué souvent de dolomies, renferme un potentiel en eau de l'ordre de 38,50 à 50 $\mathrm{hm}^{3} /$ an couvrant une superficie de $1253 \mathrm{~km}^{2}$. La nappe karstique est libre, alors que dans les compartiments effondrés de la vallée de Saïda, elle est captive.

Cette nappe karstique étudiée est localisée dans les formations 
Calcaro-Dolomitiques du Bajocien-Bathonien du secondaire dont l'épaisseur totale atteint $150 \mathrm{~m}$. Le substratum imperméable est représenté par les marnes, les argiles et les calcaires du Toarcien.

La zone d'alimentation est constituée par le plateau des Hassasnas à l'Est et le causse de Tidernatine au Sud -Est, elle est compartimentée, le compartiment le plus important en termes de débits est celui correspondant au fossé d'effondrement de Saïda. La nappe en général est en surcharge mais elle comporte une partie libre là ou les dolomies affleurent.

Plusieurs sources drainent cette nappe. Les plus importantes sont: Ain - Zerga qui est une source de trop-plein dont le débit d'étiage dans les années 1950 et même 1970 avoisinait les 100 1/s et plus ; actuellement il est de 45-50 1/s, Ain- Bent Soltane dont le débit était de 40 1/s actuellement il est à $18 \mathrm{l} / \mathrm{s}$, la source du Poirier $120 \mathrm{l} / \mathrm{s}$ nous ne disposons pas du débit actuel, mais celui-ci a certainement diminué.

Les conditions de la circulation des eaux dépendent étroitement des conditions tectoniques, celles-ci déterminant la densité et la répartition de la fissuration. Les débits des forages sont variés, les plus élevés sont obtenus à partir des forages de la région de Rebahia (Nord) ou la nappe est artésienne; l'artésianisme atteint $80 \mathrm{~m}$ et le débit 180 1/s au forage F.62.

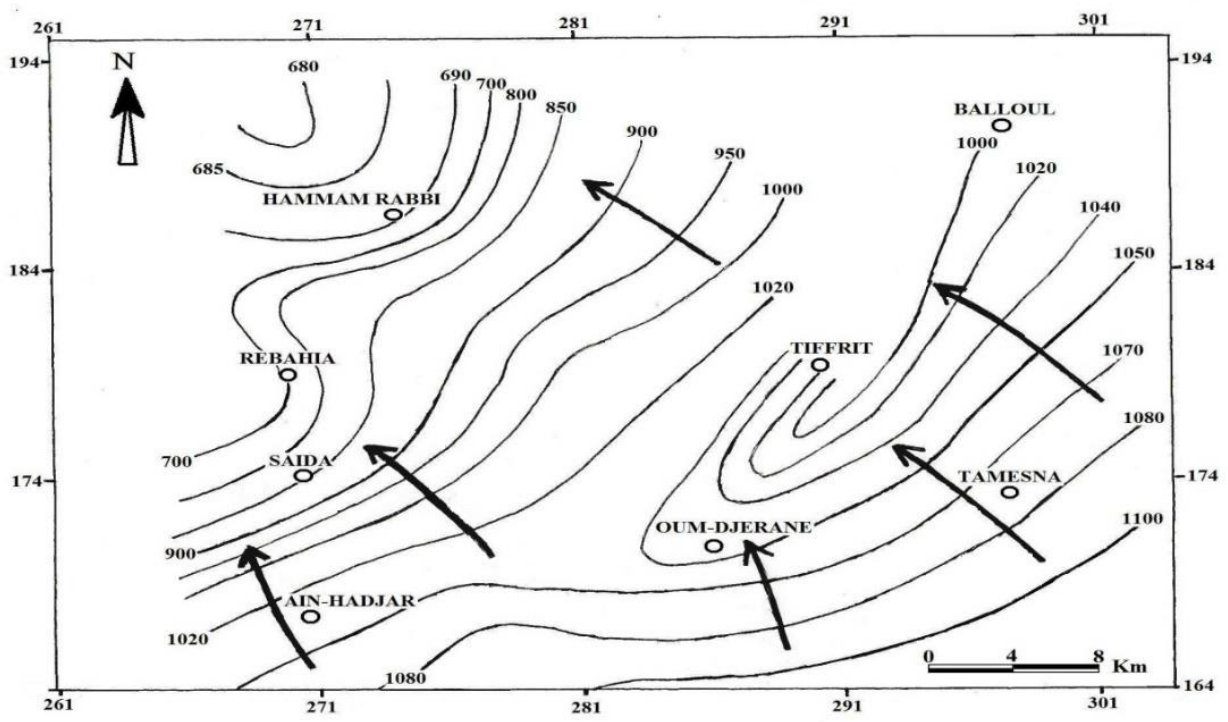

Figure 5. Carte en hydro-isohypses de la nappe karstique.

La minéralisation varie de 300 à $500 \mathrm{mg} / \mathrm{l}$ sans dépasser $600 \mathrm{mg} / \mathrm{l}$. le faciès chimique est de type carbonaté calcique et magnésien.

Cet aquifère comporte une partie libre sur le Plateau au nord-est, et une partie captive dans la région de la vallée de l'oued Saida sous les terrains plus récents du Collovo-Oxfordien et du Plio-Quaternaire. Le jeu combiné des failles à rejet et de la puissance de l'aquifère peut avoir un rôle 
important.

C'est la nappe la plus importante, dont les ressources en eau sont vraiment intéressantes pour l'alimentation des agglomérations, de l'industrie, ainsi que pour l'agriculture.

Cette nappe fait l'objet de la présente étude.

Les paramètres hydrodynamiques principaux : transmissivité et coefficient d'emmagasinement de cette nappe karstique ont été déterminés par des essais de pompage exécutés sur les forages les plus importants de la vallée de Saïda .

L'étude des aquifères situés dans les karsts, montre que la karstification entraîne l'apparition d'une organisation des écoulements. La structure qui en résulte confère à cet aquifère des caractéristiques très particulières qui le distinguent nettement des aquifères poreux et fissurés. Son approche nécessite souvent la reconnaissance préalable du degré de structuration des écoulements. L'aquifère karstique est particulièrement complexe et pose le plus de problèmes à l'hydrogéologue. Cela tient au fait que ses caractéristiques sont mal définies et que le mode d'écoulement des eaux y est tout à fait particulier Bakalowicz (1999) et Bakalowicz (2010).

Les valeurs obtenues de la transmissivité sont très variées, traduisant l'hétérogénéité hydrodynamique de l'aquifère. Ces valeurs sont de l'ordre de $1.10^{-3} \mathrm{~m}^{2} / \mathrm{s}$ en moyenne. Les fortes valeurs $\left(1.10^{-2} \mathrm{~m}^{2} / \mathrm{s}\right)$ se rencontrent plus souvent dans la vallée de Saïda dans certains forages et plus rarement sur le plateau (Nord- est). Des valeurs plus faibles, de l'ordre $1.10^{-4}$ et parfois $1.10^{-}$ 5 apparaissent dans certains forages au nord dans la vallée de Saïda. Ceci est dû à la diminution dans cette région de l'épaisseur de l'aquifère. Par conséquent toutes les valeurs peuvent être rencontrées, qui montrent une grande hétérogénéité dans l'ensemble à l'exception de quelques zones apparemment plus homogènes Les transmissivités doivent se répartir en fonction de deux facteurs particuliers :

* Degré de la karstification lié à la densité des fissures et des failles, donc à l'intensité de la tectonique.

* Epaisseur de l'aquifère suffisante en comparaison des rejets pour que les communications hydrodynamiques entre les blocs ne soient pas gênées malgré le désordre des masses carbonatées.

Les valeurs du coefficient d'emmagasinement sont de l'ordre de $1.10^{-5}$ au niveau de la nappe captive (vallée de Saida) et de $1.10^{-3}$ vers l'est, là où la nappe n'est plus artésienne mais encore en charge. Les valeurs du coefficient d'emmagasinement manquent pour la partie libre de la nappe pour des raisons techniques. Elles devraient être de l'ordre de $1.10^{-3}$ à $1.10^{-2}$.

Ici encore, il faut noter la même hétérogénéité. Les mêmes considérations peuvent être faites notamment en ce qui concerne la tectonique. 
- La région du Chott Chergui est une vaste étendue plate de 27000 $\mathrm{km}^{2}$, salée en surface et constamment humide, constituant la zone des points bas généralement à moins de $1000 \mathrm{~m}$, d'un immense Bassin hydrographique fermé de l'ordre de $49000 \mathrm{Km}^{2}$ environ portant le même nom. Il est limité, au Nord par les Monts de Saïda et de Frenda, au Sud par les Monts des Ksour, à l'Est par le Djebel Amour et à l'Ouest par le Chott El- Gharbi. Les particularités visibles de cette unité hydrogéologique complexe sont ses sources d'eau comme Ain- Skhouna et El- Kheiter, ainsi que ses paysages caractéristiques formés par les nombreuses dayas à sa bordure Nord et qui correspondraient à des cheminées ou des parties plus fissurées dans les calcaires à travers lesquelles les eaux ont plus de facilité d'infiltration. La partie centrale du Chott Chergui est très riche en ressources en eau salée, saumâtre et thermale chaude. Ce complexe renferme trois nappes aquifères intimement liées à savoir la nappe du Tertiaire continentale, la nappe du Sénonien et celle du Bajocien-Bathonien. Les calcaires et calcaires dolomitiques qui affleurent sur le flanc Sud des Monts de Saïda et dans les Monts de Mécheria, constituent l'assise perméable la plus importante de tout ce système hydrogéologique. Les calcaires du Sénonien, souvent altérés et très perméables, affleurent au Nord- Est et participent à son alimentation en donnant naissance à la source d'Ain- Skhouna.

- La région du Djebel Rémailia renferme la nappe souterraine de Rémailia qui s'étend en grande partie dans la région de Sidi Bel- Abbes. Cette formation calcaire, qui pénètre le coté Nord -Ouest de la région de Saïda dans la zone de Youb, n'a pas fait l'objet d'études approfondies et mérite donc d'être mieux connue.

\section{Elements Du Bilan}

Dans le choix du temps du bilan, c'est la périodicité qui intervient. La périodicité quasi annuelle des phénomènes météorologiques est la plus intéressante. La période n'est pas toujours rigoureusement de 12 mois. Le bilan d'un an ne se ferme pas toujours, car d'une année à l'autre les phénomènes météorologiques ne se répètent pas exactement. Cette différence imputable aux erreurs et aux variations annuelles des phénomènes autour d'une moyenne disparaît dans les bilans multi-annuels.

Dans sa formulation la plus générale le bilan hydrique s'écrit:

$$
\mathbf{P}=\mathbf{Q}+\mathbf{E T r}+\Delta \mathbf{R}(\mathbf{u}+\mathbf{h})
$$

Tout ce qui tombe $(\mathrm{P})$ dans un espace hydrologique et dans un laps de temps donné soit s'écoule (Q) soit repart dans l'atmosphère par évapotranspiration (ETr), soit participe à la recharge des réserves en eau du sol $(\mathrm{Ru})$ ou du sous-sol $(\mathrm{Rh})$.

Les variations de réserves peuvent être également négatives et contribuer aux écoulements et/ou à l'évapotranspiration. 
Le bilan hydrologique se situe donc à l'échelle du bassin (quelle que soit sa taille), considéré comme un système fermé, dont on peut résoudre l'équation de base :

$$
\mathrm{P}=\mathrm{Q}+\mathrm{ETr}+\Delta \mathrm{R}
$$

- surface totale du bassin topographique est de $1253 \mathrm{~km}^{2}$.

- Surface total de l'impluvium karstique : $723 \mathrm{~km}^{2}$.

- Surface total de l'impluvium très peu perméable : $530 \mathrm{~km}^{2}$.

\section{Les précipitations et l'évapotranspiration}

Le réseau d'observation de la région est constitué de 31 pluviomètres et deux pluviographes. Ces stations étaient toutes défaillantes (les séries présentent des lacunes) sauf la station météorologique de Saida qui présente une série continue et homogène, la moyenne des précipitations mesurée sur une période de 25années ( 1975 - 2000) donne $335.6 \mathrm{~mm}$.

Dans des études antérieures qui concernent la région, Seltzer (1946) indique que la moyenne des précipitations annuelles pour la période 19131938 est de l'ordre de $450 \mathrm{~mm}$.

Cette moyenne des précipitations annuelle enregistre, comparativement à la période de

1975- 2000, un déficit de l'ordre de $114.4 \mathrm{~mm}$.

Ce déficit ainsi enregistré, a été soulevé par d'autres auteurs qui ont aussi relevés l'aspect de fluctuations climatiques et la tendance de l'aridité du climat de la région Meddi et Meddi (2009) ; Meddi et Hubert (2003); Laborde (1993).

Tableau 1. Précipitations Moyennes Mensuelles à la station de Saida $(1975$ - 2000)

\begin{tabular}{|c|c|c|c|c|c|c|c|c|c|c|c|c|c|}
\hline Mois & Sep & Oct & Nov & Déc & Jan & Fév & Mar & Avr & Mai & Juin & Juil & Aout & Année \\
\hline $\begin{array}{c}\text { Précipitations } \\
(\mathrm{mm})\end{array}$ & 15.6 & 36.6 & 33.5 & 36.8 & 38.8 & 37.9 & 46.6 & 37.3 & 26.5 & 12.4 & 3.4 & 10.2 & 335.6 \\
\hline
\end{tabular}

L'alimentation de la nappe karstique se réalise uniquement par l'infiltration des eaux atmosphériques (apport pratiquement unique: les précipitations).

Les zones d'alimentation de cette nappe se situent à l'Est de Saïda, dans les causses de Tidernatine et au plateau des Hassasnas ou les dolomies et calcaires du Bajocien-Bathonien affleurent très largement. L'infiltration dans cette région doit-être importante et rapide.

La zone d'évaporation est réduite, l'infiltration devrait être ici bien supérieure à 10\% d'après Grandarovski (1963).

L'évaluation de l'infiltration d'un bassin à l'autre de l'Ouest vers l'Est parait être assez bien liée à la tectonique, ou du moins à la karstification qui en a résulté et cette karstification doit diminuer d'une façon générale d'Ouest en Est au fur et à mesure que l'on s'éloigne du fossé d'effondrement de la vallée de Saïda et de la zone tectonique Saïda - Tiffrit. 
La quantité d'eau qui s'infiltre dans la nappe correspond au débit total des exutoires.

Le bilan de la nappe est pratiquement fermé c'est-à-dire : $\mathrm{I}=\mathrm{Q}$ total .

Pour $\mathrm{P}=335.6 \mathrm{~mm} \rightarrow \mathrm{I}=1449 \mathrm{l} / \mathrm{s}$ qui est égal au débit total des sources + forages

Pour $\mathrm{P}=450.0 \mathrm{~mm} \rightarrow \mathrm{I}=1847 \mathrm{l} / \mathrm{s} \quad$ (Voir Tableau 6). $723 \mathrm{~km}^{2}$.

La surface affleurante du réservoir (Dolomies et calcaires) est égale à

Une pluviométrie moyenne de $\mathrm{P}=450 \mathrm{~mm}$ correspond à un volume d'eau de $325.3 \mathrm{hm}^{3} / \mathrm{an}$.

Une pluviométrie moyenne de $\mathrm{P}=335.6 \mathrm{~mm}$ correspond à un volume d'eau de $242.6 \mathrm{hm}^{3} / \mathrm{an}$.

Le coefficient d'infiltration $\mathrm{K}_{\mathrm{I}}=\mathrm{I} / \mathrm{P}$

Pour un coefficient d'infiltration élevé les précipitations peuvent en s'infiltrant plus facilement se mettre rapidement à l'abri de l'évapotranspiration sous la tranche sèche de l'aquifère karstique.

La Région d'Oum -Djerane au Centre du plateau d'alimentation avec la présence de nombreux «Rhar » (Cavernes) comme celui du Rhar Amira doit relever considérablement le coefficient d'infiltration du bassin de Saïda qui peut atteindre $25 \%$ selon le Bureau d'étude Bininal Water Engineering (2012).

Le calcul de l'évapotranspiration a été fait par La méthode de Thorthwaite. Les calculs ont été exécutés sur la base des données météorologiques qui correspondent notamment à une température moyenne annuelle de $17.9^{\circ}$. Pour les terrains karstiques du jurassique inférieur et moyen (dolomies et calcaires) la réserve d'eau utile à la végétation est estimée à $0 \mathrm{~mm}$ (humidité de rétention et frange capillaire inexistantes).

L'évapotranspiration réelle calculée donne une valeur égale à 273 $\mathrm{mm}$ qui correspond à $82 \%$ des Précipitations. L'évapotranspiration sur l'impluvium dolomitique est considérable, ce terme est évalué par différence entre la pluviométrie et l'écoulement souterrain, le ruissellement superficiel étant négligeable.

\section{Ruissellement Superficiel et Ecoulement Souterrain}

L'écoulement est entièrement souterrain. Ceci est visible sur le terrain, il n'y a pas de décomposition à exécuter Blavoux et Mudry (1983) ; Bakalowicz (1979).

Les parties géologique et hydrogéologique montrent de façon plus détaillée que pour les terrains carbonatés karstifiés du jurassique inférieur et moyen le ruissellement superficiel est négligeable et le ruissellement souterrain est important Bakalowicz (2005) ; Bakalowicz et Mangin (1980).Ce ruissellement souterrain est à l'origine de la majorité des sources 
karstiques de la région.

Un seul oued non pérenne coule en terrain dolomitique, mais surtout en période de crue. Il prend sa source à Ain-Foufot, dont il porte le nom et rejoint Ain-Tiffrit la piézométrie ainsi que l'aspect relativement limpide de l'eau montre que cet écoulement correspond entièrement au drainage de l'aquifère karstique.Tout cet écoulement est mesuré par la station hydrométrique de Tiffrit.

Nous rappelons que l'impluvium dolomitique pour l'ensemble de tous les bassins est limité par les contacts avec les affleurements des terrains détritiques d'une part et d'autre part par les lignes à la fois topographiques et piézométriques de partage des eaux du Sud et de l'Est.

Avant la karstification les surfaces affleurantes dolomitiques étaient soumises à un ruissellement superficiel important avec fonction de bassins versants topographiques à la faveur de phénomènes d'érosion et de dissolution superficiels. Ensuite une tectonique cassante a pu intervenir compte tenu de la nature de ces terrains carbonatés. Des failles se sont constituées de façons tout à fait indépendantes de la formation des bassins versants topographiques encore fonctionnels. Ces failles ont favorisé une karstification liée aux lignes de force tectoniques. Cette karstification s'est développée de plus en plus en profondeur favorisant l'infiltration et la constitution d'une nappe dont le sens d'écoulement est également indépendant de la formation des bassins versants. Actuellement ces bassins versants ne sont presque plus fonctionnels car la plus grande partie de l'infiltration doit absorber l'eau qui ruisselait lorsque la dolomie était compacte. Cette explication est évidemment très schématique et ne fait pas intervenir les autres terrains plus récents et non carbonatés qui au cours de ces phénomènes ont été décapés également par l'érosion.

La carte piézométrique présentée donne la réponse à toute une série de questions importantes concernant la circulation et la répartition des eaux souterraines de la nappe karstique.

L'écoulement souterrain est orienté en général Sud est - Nord ouest.

La ligne principale de partage des eaux souterraines suit approximativement la limite topographique Sud des bassins versants; les hauteurs de Djebel Sidi Youssef, Djebel El Yhoudia, Djebel El Leba.

Au sud de cette ligne de partage, l'écoulement souterrain est dirigé vers la plaine des Mâalif ; Khalfallah et la dépression du Chott Chergui plus loin ce qui n'est pas visibles sur cette carte.

$\mathrm{Au}$ Nord, le flux souterrain est réparti en plusieurs bassins hydrogéologiques délimités par des lignes de partage des eaux. Il y en a quatre :

- Bassin hydrogéologique de Saïda avec deux sous-bassins Rebahia et Hammam Rabi. 
- Bassin hydrogéologique de Tiffrit.

- Bassin hydrogéologique du plateau d'Ain- Soltane avec deux sousbassins (d'Ain Soltane et

de Sidi- Yahia).

- Bassin hydrogéologique du plateau d'Ain Balloul.

\section{Les Sources}

La vidange de la nappe karstique se réalise exclusivement par les sources. Deux zones de décharge se distinguent :

- Zone Ouest drainant le bassin hydrogéologique de Saïda.

- Zone Nord drainant les trois autres bassins hydrogéologiques de Tiffrit, d'Ain Soltane et de Balloul d'après Boudjemaa (2006).

Outre les sources se trouvant dans les zones citées, il existe encore des lignes de décharge diffuses (suintement) dont le débit est mesurable uniquement à la base des jaugeages différentiels sur le tronçon correspondant. Dans le tableau 2 nous présentons la liste des sources karstiques de la région.

Tableau 2. Liste des sources karstiques au niveau de la région

\begin{tabular}{|c|c|}
\hline BASSIN HYDROGEOLOGIQUE & SOURCES \\
\hline $\begin{array}{l}\text { Bassin Hydrogéologique } \\
\text { de Saida }\end{array}$ & $\begin{array}{l}\text { Ain -Zerga, Source de Poirier, Ain -Moussoualuf, Ain } \\
\text {-Ben Soltane, Source Ronde, Ain -Fakhroun, Source } \\
\text { Tiède, Source de la Tranchée, Ain -Hadjar, Ain } \\
\text { Tagourarelt, Ain -Tameshoum, Ain -beida, Ain- Said } \\
\text { Fatah, Ain -Touta, Ain -Es Srhirat, Ain -Mathnia, Ain } \\
\text { Messaoud, Ain -Karma, Ain - Fakroun et autres petites } \\
\text { sources (noms inconnus). }\end{array}$ \\
\hline $\begin{array}{c}\text { Bassin Hydrogéologique } \\
\text { de Tiffrit }\end{array}$ & Tiffrit, Toufout, autres petites sources (noms inconnus). \\
\hline $\begin{array}{l}\text { Bassin Hydrogéologique } \\
\text { du Plateau d'Ain -Soltane }\end{array}$ & $\begin{array}{l}\text { Ain -Yahia, Ain -Sultane, Ain- M'Nir, Ain -Fertas, Ain- } \\
\text { Kerma et petites sources voisines, Station Sidi Yahia. }\end{array}$ \\
\hline $\begin{array}{l}\text { Bassin Hydrogéologique } \\
\text { du Plateau d'Ain Balloul }\end{array}$ & $\begin{array}{l}\text { Ain- Bou Amar, Ain- Balloul, Ain- Seridj, Ain Souig, } \\
\text { Ain- Ras Guersif, Ain -Mizraf, Ain- Tefaha, Ain El - } \\
\text { Hadjel et d'autres sources et suintements. }\end{array}$ \\
\hline
\end{tabular}

La plupart de ces sources ont un débit moyen compris entre 1 à 10 1/s. cependant, il existe quelques unes dont le débit est très important telles que les sources de Ain - El Zerga, Ain -Soltane, Source du Poirier, Ain Fakroun et Ain - Tiffrit.

Dans le Tableau 3 les débits totaux des sources des différentes zones correspondent aux périodes ayant respectivement une pluie moyenne $\mathrm{P}=$ $335.6 \mathrm{~mm}$ et $\mathrm{P}=450 \mathrm{~mm}$. 
Tableau 3. Valeurs de l'écoulement souterrain aux sources

\begin{tabular}{|c|c|c|c|c|c|c|}
\hline $\begin{array}{c}\text { Stations } \\
\text { Hydrométriques }\end{array}$ & \multicolumn{3}{|c|}{$\begin{array}{l}\text { Oued Saïda à } \\
\text { PK 50 }\end{array}$} & $\begin{array}{l}\text { Hasna et } \\
\text { Guernida }\end{array}$ & $\begin{array}{l}\text { Tiffrit }- \text { Sidi } \\
\text { Mimoune }\end{array}$ & $\begin{array}{c}\text { Ensemble des } \\
\text { Stations }\end{array}$ \\
\hline $\begin{array}{c}\text { Bassins } \\
\text { Hydrogéologiques }\end{array}$ & \multicolumn{3}{|c|}{$\begin{array}{c}\text { Bassin de } \\
\text { Saida-Eaux Chaudes }\end{array}$} & $\begin{array}{c}\text { Bassin de } \\
\text { Ain- } \\
\text { Soltane }\end{array}$ & $\begin{array}{c}\text { Bassin de } \\
\text { Tiffrit+Balloul }\end{array}$ & $\begin{array}{c}\text { Ensemble des } \\
\text { Bassins }\end{array}$ \\
\hline $\begin{array}{c}\text { Surface impluvium } \\
* \text { totale }\left(\mathrm{km}^{2}\right) \\
* \text { dolomitique }\left(\mathrm{km}^{2}\right) \\
* \text { détritique }\left(\mathrm{km}^{2}\right)\end{array}$ & \multicolumn{3}{|c|}{653} & $\begin{array}{l}114 \\
42 \\
72\end{array}$ & $\begin{array}{c}486 \\
400 \\
86\end{array}$ & $\begin{array}{l}1253 \\
723 \\
530\end{array}$ \\
\hline Zônes & I & II & III & IV & $\mathrm{V}$ & \\
\hline \multirow{4}{*}{$* \mathrm{P}=335.6 \mathrm{~m}$} & \multicolumn{6}{|c|}{ Ecoulement Souterrain par les Sources $(\mathrm{l} / \mathrm{s})$} \\
\hline & \multirow{3}{*}{$\begin{array}{l}61 \\
82\end{array}$} & \multirow{3}{*}{$\begin{array}{l}540 \\
724\end{array}$} & \multirow{3}{*}{$\begin{array}{l}59 \\
79\end{array}$} & \multirow{3}{*}{$\begin{array}{c}86 \\
115\end{array}$} & \multirow{3}{*}{$\begin{array}{l}424 \\
568\end{array}$} & $\begin{array}{c}\text { Ensemble des } \\
\text { Zones }\end{array}$ \\
\hline & & & & & & 1170 \\
\hline & & & & & & 1568 \\
\hline
\end{tabular}

Le débit total souterrain de l'aquifère est égal au débit des sources augmenté du débit extrait par forages et puits.

\section{Forages et puits}

Pour les prélèvements de ses ressources en eau souterraines locales, la région de Saïda est passée de 675 points d'eau exploités en 2003 à 1166 points d'eau en 2004 comprenant :

67 forages et 3 sources pour l'alimentation en eau potable, 455 forages, 343 puits et 301 points de prélèvements pour l'irrigation (Source Direction des ressources en eau de Saida).

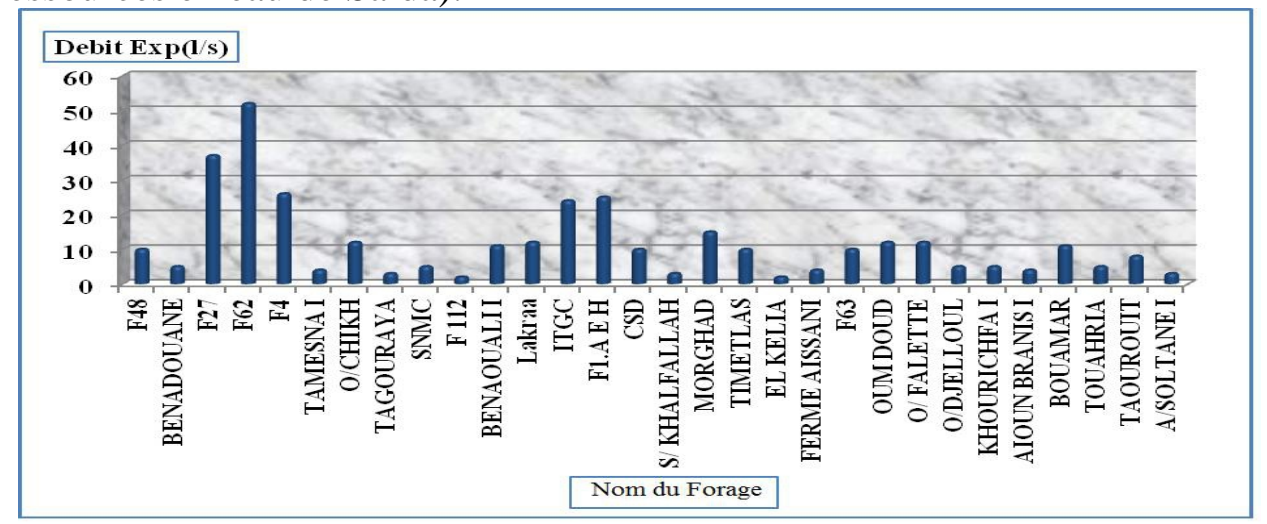

Figure 6. Histogramme correspondant aux débits d'exploitation des forages de la région.

L'Aquifère du Bajocien-Bathonien est karstique et constituait le réservoir essentiel pour l'alimentation en eau potable de la ville de Saïda. Cet aquifère qui est libre dans les régions où les dolomies et calcaires affleurent (plateau d'Oum- Djerane et Tidernatine, à l'Est de la ville) devient captif lorsqu'il est surmonté par le Collovo-Oxfordien, argilo-gréseux, qui constitue le toit de cette nappe. 


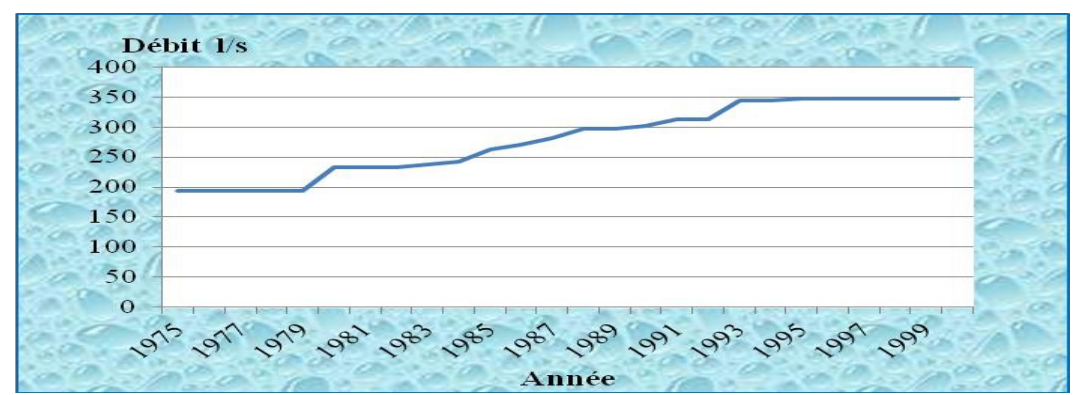

Figure 7. Courbe de l'évolution des débits d'exploitation des forages de la nappe karstique sur la période $1975-2000$.

Parmi les forages existants, il n'y a que 30 forages en exploitation permanente pour notre période d'étude.

Tableau 4. Valeurs des débits d'exploitation des forages de la nappe karstique

\begin{tabular}{|c|c|c|c|c|}
\hline $\begin{array}{c}\mathrm{N} \\
\mathrm{o}\end{array}$ & Nom du Forage & $\begin{array}{c}\text { Débit } \\
\text { d'exploitation } \\
(1 / \mathrm{s})\end{array}$ & $\begin{array}{l}\text { Date de } \\
\text { Réalisation }\end{array}$ & $\begin{array}{l}\text { Utilisation des Eaux } \\
\text { AEP/IND./IRR }\end{array}$ \\
\hline 1 & F.48 & 10 & 1968 & IND \\
\hline 2 & Benadouane & 5 & 1983 & AEP \\
\hline 3 & F.27 & 37 & 1972 & AEP/IND \\
\hline 4 & F.62 & 52 & 1972 & AEP/IND \\
\hline 5 & F.4 & 26 & 1973 & AEP \\
\hline 6 & Tamesna 1 & 4 & 1964 & AEP \\
\hline 7 & $\begin{array}{l}\text { Oued Chikh (Sidi- } \\
\text { Gacem) }\end{array}$ & 12 & 1971 & AEP \\
\hline 8 & Tagouraya & 3 & 1989 & AEP \\
\hline 9 & SNMC & 5 & 1980 & IND \\
\hline 10 & F. 112 & 2 & 1975 & AEP \\
\hline 11 & $\begin{array}{l}\text { Benaouali I (Sidi- } \\
\text { Ghiat) }\end{array}$ & 11 & 1968 & AEP \\
\hline 12 & Lakraa & 12 & 1967 & AEP \\
\hline 13 & I.T.G.C. & 24 & 1993 & AEP \\
\hline 14 & F1. Ain-El-Hadjar & 25 & 1980 & AEP/IND \\
\hline 15 & C.S.D. & 10 & 1980 & IND \\
\hline 16 & Sidi - Khalfallah & 3 & 1988 & AEP \\
\hline 17 & Morghad & 15 & 1969 & AEP \\
\hline 18 & Timetlas & 10 & 1991 & AEP \\
\hline 19 & El-kelia & 2 & 1969 & AEP \\
\hline 20 & Ferme Aissani & 4 & 1985 & AEP/IRR \\
\hline 21 & F63 & 10 & 1970 & AEP \\
\hline 22 & Oum - Doude & 12 & 1987 & AEP \\
\hline 23 & Oued Falette & 12 & 1988 & AEP \\
\hline 24 & Ouled Djelloul & 5 & 1990 & AEP \\
\hline 25 & Khourichfa 1 & 5 & 1984 & AEP \\
\hline 26 & Aioun- Branis 1 & 4 & 1985 & AEP \\
\hline 27 & Bouamar & 11 & 1985 & AEP \\
\hline 28 & Touahria & 5 & 1986 & AEP \\
\hline 29 & Taourouit & 8 & 1993 & AEP \\
\hline \multirow[t]{2}{*}{30} & Ain- Soltane 1 & 3 & 1995 & AEP \\
\hline & Total débits & 347 & & \\
\hline
\end{tabular}

Source : Direction des ressources en eaux de Saida. (AEP: Alimentation en eau potable ;

IND : Industrielle ; IRR : Irrigation). 
Comme les forages sont mis en exploitation en des dates différentes, le calcul de la moyenne des débits d'exploitation s'impose. Cette moyenne sur la période 1975 -2000 est égale à 279 1/s.

\section{Débit total de l'écoulement souterrain}

Le débit souterrain de toute cette région est constitué par l'écoulement dans l'aquifère karstique, les autres aquifères ayant un écoulement souterrain propre relativement négligeable. Le chiffre obtenu pour le débit total des sources est calculé pour des précipitations moyennes de $335.6 \mathrm{~mm}$ correspondant à la période d'étude et aussi pour des précipitations de $450 \mathrm{~mm}$. A ce débit s'ajoute le débit extrait par forages et puits (tableau 5).

Tableau 5. Débit total de l'écoulement souterrain de la nappe karstique sur 25 années.

\begin{tabular}{|c|c|c|}
\hline Pluviométrie (mm) & 335.6 & 450 \\
\hline débit total des sources $(1 / \mathrm{s})$ & 1170 & 1568 \\
\hline débit extrait par forages et puits $(1 / \mathrm{s})$ & 279 & 279 \\
\hline Débit total souterrain $(1 / \mathrm{s})$ & 1449 & 1847 \\
\hline
\end{tabular}

\section{Calcul du bilan de la nappe}

A la base du tableau 3 pour $\mathrm{P}=335.6 \mathrm{~mm}$ le chiffre $1170 \mathrm{l} / \mathrm{s}$ représente la presque totalité du débit de l'aquifère aux sources à l'exception du débit extrait par forages et puits qui correspond lui à un total de 279 1/s.

Sur une longue période de temps (moyenne sur plusieurs années) l'équation du bilan peut s'écrire de la façon suivante :

Pluie $(\mathrm{P})=$ Ecoulement Souterrain $\left(\mathrm{R}_{\mathrm{n}}\right)+$ Ecoulement Superficiel $\left(\mathrm{R}_{\mathrm{s}}\right)+$ Evapotranspiration $(\mathrm{ETr})$

\pm Echanges avec la Nappe Superficielle $(\mathrm{E}) \pm$ Variation des Réserves $\left(\mathrm{V}_{\mathrm{r}}\right)$

L'équation se résout après élimination des termes négligeables sur une longue période de temps à :

$$
P=R n+E T r
$$

Avec $R n$ : débit total des sources + débit extrait par forages et puits.

$$
E T r=P-R n
$$

Pour résumer et d'après le tableau récapitulatif du bilan, on remarque que pour les deux périodes de temps 1913-1938 et 1975- 2000 les réserves souterraines de la nappe ont diminuées de $21.6 \%$, une différence de 57.4 $45=12.4 \mathrm{Hm}^{3}$, chiffre assez important. Les différents éléments du bilan sont résumés dans le tableau 6 . 
Tableau 6. Eléments et bilan de la nappe Karstique

\begin{tabular}{|c|c|c|c|c|c|c|c|c|c|c|}
\hline \multicolumn{2}{|c|}{ Pluie P } & $\begin{array}{l}\text { Impluvium } \\
\text { dolomitique }\end{array}$ & \multicolumn{3}{|c|}{\begin{tabular}{c}
\multicolumn{2}{|c|}{ Ruissellement souterrain } \\
total Rn
\end{tabular}} & $\begin{array}{l}\text { Coefficient } \\
\text { de } \\
\text { l'écoulement }\end{array}$ & $\begin{array}{l}\text { Evapotranspira- } \\
\text { tion réelle ETr }\end{array}$ & $\begin{array}{c}\text { Débit } \\
\text { souterrain } \\
\text { spécifique }\end{array}$ \\
\hline $\mathrm{mm}$ & $\mathrm{Hm}^{3}$ & $\mathrm{Km}^{2}$ & $\mathrm{~mm}$ & $\mathrm{Hm}^{3}$ & $1 / \mathrm{s}$ & $\begin{array}{c}\% \\
\mathrm{de} \mathrm{P}\end{array}$ & $\mathrm{Ki}=\mathrm{I} / \mathrm{P}$ & $\mathrm{mm}$ & $\% \mathrm{de} \mathrm{P}$ & $1 / \mathrm{s} / \mathrm{Km}^{2}$ \\
\hline 335.6 & 242.6 & 723.0 & 62.3 & 45.0 & 1449 & 18.6 & 0.18 & 273.3 & 81.4 & 2.0 \\
\hline 450.0 & 325.3 & 723.0 & 79.4 & 57.4 & 1847 & 17.6 & 0.17 & 370.6 & 82.3 & 2.5 \\
\hline
\end{tabular}

\section{Conclusion}

Ce travail nous a permis d'avoir une vue sur l'hydrogéologie de la région de Saida ou est située la nappe karstique qui présente une grande importance pour la région car elle alimente en eau la population et les unités économiques.

La comparaison des séries pluviométriques récentes et anciennes montre que la moyenne des précipitations a connue une baisse ces dernières décennies (période 1913-1938 et 1975 - 2000), cette baisse avoisine les $25 \%$ pour la région de Saida qui se caractérise par un climat semi-aride.

Cette fluctuation climatique s'est répercutée négativement sur les réserves en eau des nappes souterraines qui sont alimentées principalement par les précipitations.

L'écoulement aux sources qui était de 1568 1/s est passé à 1170 1/s et certaines sources se sont asséchées.

Les résultats de notre étude sur le bilan de la nappe souterraine de Saida montrent que les réserves sont passées de $57.4 \mathrm{Hm} 3$ à 45 hm 3 sur les deux périodes d'étude. Face à des ressources en eau qui deviennent de plus en plus limitées et une demande en eau croissante par les différents secteurs utilisateurs en plein essor et de surcroit dans une région semi-aride où la sécheresse persiste depuis des décennies, il est nécessaire de mettre en place un schéma d'exploitation plus efficace pour une gestion optimale et rigoureuse à long terme des ressources en eau de cette nappe.

\section{References:}

Bakalowicz M., (1979. Contribution de la géochimie des eaux à la connaissance de l'aquifère karstique et de la karstification. Doctorat ès sciences naturelles, université P.et M. Curie, Paris.

Bakalowicz M. et A.Mangin (1980). L'aquifère karstique. Sa définition, ses caractéristiques et son identification. Mémoire hors série, Soc. géol. France 11,71-79.

Bakalowicz M. (1999). Guide technique $\mathrm{n}^{\circ} 3$, connaissances et gestion des ressources en eau souterraine dans les régions karstiques. AERM and $C$. 
Bakalowicz M. (2005). Karst groundwater, a challenge for new ressources. Hydrogéology Journal 13, 148-160.

Bakalowicz M. (2010). Karst et ressources en eau souterraine: un atout pour le développement des pays Méditerranéens. Sécheresse 21 (4), 319-22.

Blaboux B.et J. Mudry (1983). Séparation des composantes de l'écoulement d'un exutoire karstique à l'aide des méthodes physico-chimiques. Hydrogéologie-Géologie de l'ingénieur. B.R.G.M, Orléans, 269-278.

Boudjemaa M.(2006). Contribution à l'étude de l'impact de la pollution de l'oued de Saïda sur la qualité des eaux minérales. Thèse de Magister en hydraulique, université de Mascara, Algérie.

Bureau d'étude Bininal Water Engineering, (2012). Actualisation de l'étude hydrogéologique de la vallée de Saida. Rapport d'étude, Direction des ressources en eau, Saida.

Decamps P. (1973). Etude hydrogéologique de la région de SaidaTagrémaret.État des connaissances et des études en cours. Rapport S.E.S .Saida .

Grandarovski G. (1963). Etude hydrogéologique de la région de Saida. Rapport d'étude, Direction des ressources en eau, Saida.

Laborde J.P. (1993). Carte pluviométrique de l'Algérie du Nord à l'échelle $d u$ 1/500 000. Notice explicative. Projet PNUD/ALG/88/021, Alger, Agence nationale des ressources hydrauliques.

Meddi M.et P. Hubert (2003). Impact de la modification du régime pluviométrique sur les ressources en eau du nord-ouest de l'Algérie. Hydrology of the Mediterranean and semiarid Regions. IAHS Publications 278.

Meddi H.et M. Meddi (2009).Variabilité des précipitations annuelles du Nord Ouest de l'Algérie. Sécheresse 20,1, 57-65.

Pitaud G. (1973). Etude hydrogéologique pour la mise en valeur de la vallée d'oued de Saida. Paris, Presse Universitaire France.

Seltzer P. (1946). Le climat de l'Algérie. Travaux d'Institut de Météorologie et de Physique du Globe. Université d'Alger, Alger, 219 p. 